\title{
Multi-objective Optimization Method for Radiation Noises in Cylinder Structures under Mixed Excitation
}

\author{
Jie Gao ${ }^{\text {a }}$, Ning Qiang, ${ }^{\text {, }}$ \\ College of Physics and Information Technology, Shaanxi Normal University, Shaanxi, Xian 710119, \\ China \\ agaojie@snnu.edu.cn bqn315@snnu.edu.cn
}

Keywords: Structure-borne acoustic radiation, Multi-objective optimized, Intermediate frequency region

\begin{abstract}
The multi-objective optimization design for complicated reinforced cylinder structures was discussed by combining the ISIGHT multidisciplinary optimization design framework. Firstly, the mathematical model of acoustic radiation optimization of cylinder structures was established for sound and vibration analysis of simulation objects, thus determining the optimal design parameters. Secondly, software frameworks were integrated and the multi-objective optimization process was designed based on the ISIGHT platform. Finally, iterative computations were carried out by the optimization platform. This is the end of optimization operation. Results demonstrated that acoustic characteristics of the reinforced cylinder shell structures are improved to some extent after size optimization. The optimization is fruitful.
\end{abstract}

\section{Introduction}

Noise problems in structural cavity are actually the vibration and acoustic radiation problems of complicated shell. Compared with common structural optimization, research on acoustic radiation optimization of structures is more complicated[1,2,3]. This is mainly because the optimization process involves not only structural problems, but also the coupling relationship between acoustic radiation and the structure. This is a typical multi-disciplinary design optimization (MDO) problem[4,5,6]. In acoustic optimization model of structures, acoustic characteristics of the structure are often used as the objective function or constraint conditions of optimization, while structural volume, shape or topological structure are used as the modification objects in for optimization analysis. Compared with common structural dynamic response optimization, constraints in acoustic radiation optimization of structures also may include structural response parameters which are desired by designers[7,8,9,10].

In this study, multi-objective optimization was designed by using iSIGHT multi-disciplinary integrated platform as the software framework and the integrated sound and vibration computing software VA One. VAOne and iSIGHT were connected by the programming language VC++. The computing framework of multi-disciplinary optimization design of intracavity noise in the complicated reinforced cylinder structure was constructed by using the FE-SEA hybrid computing method as the theoretical basis. The multi-objective optimization of intracavity noise was carried out by using geometric size of the structure as the design variable, structural dynamic response analysis and sound field analysis as sub-disciplines, and total structural mass ad average intracavity sound pressure level as constraint and objective function, respectively.

\section{Optimization design strategy of acoustic radiation in structural cavity}

Multi-discipline design optimization of structural acoustic radiation uses acoustic indexes as objective function or constraint. The objective function can be field point sound pressure, structural acoustic radiation power and structural mass, or the multi-objective function formed by weighting of these indexes. The mathematical model of multi-objective minimization can be described as:

min: $\quad F(x)=\left(F_{1}(x), F_{2}(x), \cdots, F_{m}(x)\right)$ 


$$
\begin{array}{ll}
\text { s.t. } & g_{i} \leq 0, \quad i=1,2 . ., p \\
& h_{j}(\mathrm{x})=0, \quad j=1,2 . ., q \\
& x_{1} \leq x_{i} \leq x_{n} i=1,2 . ., n
\end{array}
$$

where $F(x)$ is the objective function of vectors and $F(x)(i=1,2 . ., m)$ is the sub-objective functions. $g_{i} \leq 0$ is an inequality constraint, $h_{j}(\mathrm{x})=0$ is an equality constraint, and $x_{i}$ is the constraint of design variables. Multi-discipline optimization problem of structural acoustic radiation is to search on group of design variable in the design space domain to meet the preset objective function and constraint conditions no matter what kind of optimization model is used. Since structural acoustic radiation optimization involves multi-discipline coupling factors and it is one multi-objective optimization problem, full considerations shall be given to factors of other disciplines when determining the objective function and constraint conditions. It is suggested to establishing the mathematical optimization model from the global system. After the appropriate mathematical model is established, it is able to make specific numerical simulation analysis.

In this study, noise optimization in structural cavity aims to minimize acoustic responses indexes (e.g. average sound pressure level) in structural cavity by modifying the structural design parameters. Therefore, the following three problems have to be solved during the acoustic radiation optimization process: 1. Calculating acoustic responses of the structural system to external exciting force, i.e., acoustic radiation response indexes. 2. Calculating impact degree of acoustic response indexes on structural design parameters, which provides directions for structural modification. 3 . Appropriate search strategy. This means to choose optimization algorithm and optimization program.

\section{Integration of software framework and optimization design flow}

Sound and vibration analysis module of the structure is an important component in the whole software design framework. How iSIGHT integrates VA One to realize automatic analysis and solving is one of key problems that have to be solved. In this study, multi-objective optimization was designed by using iSIGHT multi-disciplinary integrated platform as the software framework and the integrated sound and vibration computing software VA One. VAOne and iSIGHT were connected by the programming language VC++. During engineering design using iSIGHT, the first thing is to integrate multiple links in the design. This is easy to realize mutual calling and integration of multiple disciplines. In this optimization problem, it is necessary to make secondary development of VA One firstly before integrating VA One in the iSIGHT.

Secondary development of VA One. API language of VA One provides interface of development instruments and software to $\mathrm{C}++$ dynamic link library. All data can be output as data files which are easy to be read by other software. The secondary development software was programmed by Visual $\mathrm{C}++$ to realize integration of VA One and iSIGHT. The programmed secondary development software can realized following functions: 1 . it can read any parameters in database in VA One computing model and output variable name and variable value into text file. 2. It can read sound pressure level response value of vocal cavity subsystem in the outcome file of VA One and output the vocal cavity subsystem name and sound pressure level value into text file. SEA Matrix as well as intermediate results of power inputs and outputs were extracted and stored in textile documents. 3. The A One computing model database can be modified according to input of text files and the modified model was used in new computations.

Here, a friendly user interface for cavity structures which need size optimized design was programmed by Visual $\mathrm{C}++$. It can set the background to open the model and set thickness of various substructures and types of vocal cavities. Next, all data information were input into the programmed computing software SPLSolver.exe to calculate sound pressure level of vocal cavity. Finally, computing results were output to text files, which is conducive for recall of iSIGHT. 
Integration process of iSIGHT and VA One. This calculation process is in iSIGHT. It realizes the goal model modification by changing information of design variables in input.txt. The new model after parameter modification can execute SPLSolver.exe to solve the problem. Finally, computing results are output and new result text (output.txt) is produced. New computing results of average sound pressure level were read from iSIGHT. Later, whether this result is the optimal solution was evaluated by the optimization algorithm of iSIGHT. If not, continue the next iteration until find the optimal results - the lowest sound pressure level. The corresponding input parameters are the optimal design parameters. Data flow direction in iSIGHT is shown in Fig.1.

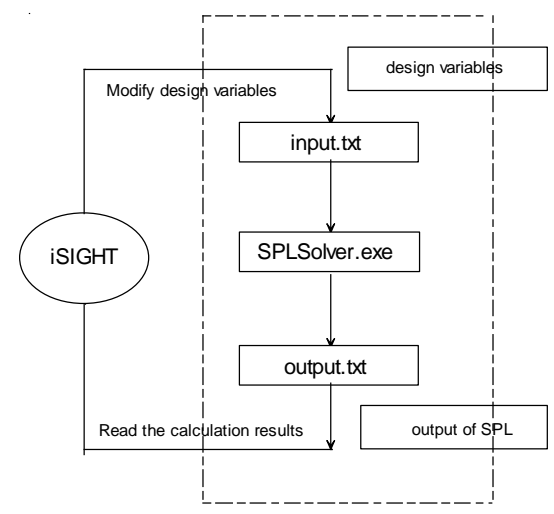

Fig.1 Data transmission in iSIGHT

After integration of VA One and iSIGHT and the establishment of optimization task, it also has to design the optimization scheme in the iSIGHT platform and set design process variables, such as objective function, constraint, design variables and optimization algorithms. Finally, execute the final optimization calculation and output the optimal design variable.

Parameter setting in the structural optimization model. Variable design, constraint conditions, objective function and optimization algorithm are several basic elements in optimizing the mathematical model of the problem. Firstly, choose appropriate variables according to contribution rates of substructures to sound pressure level in the structural cavity, or there are excessive design variables to increase computing load and influence computing speed. Therefore, parameters of several substructures with the largest contributions to sound pressure level in the structural cavity are chosen as design variables.

Here, upper and lower limits of design variables were designed with considerations to size constraint and total mass constraint. The total mass constraint was determined that changes are less than $10 \%$ of initial mass. Generally speaking, structural optimization also has to consider strength constraint, which hasn't been considered here. Since this chapter emphasizes on sound and vibration response features of optimized structure, many analysis types will make the optimization flow over complicated and low optimization efficiency. The objective function of this optimization problem is to the optimization of total structural mass and intracavity sound pressure level.

Computing modules on the iSIGHT include sound pressure level computing module, mass computing model and weighted computing module. The sound pressure level computing module mainly calculates sound pressure level in the structural cavity, which has to recall the VAOne.exe computing program in the integrated platform. The mass computing module calculates total mass of the structure during the optimization iteration. The weighted computing module is for weighted computing of optimization results of above two modules, finally getting the multi-objective optimization results. The multi-objective optimization design in ISIGHT is shown in Fig.2. 


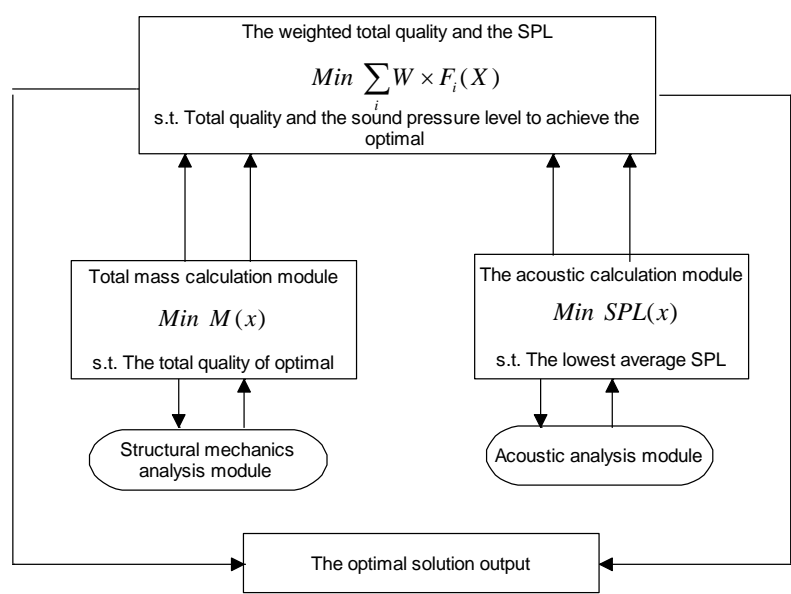

Fig. 2 Multi-objective optimization design framework.

According to two objective functions which to be realized in this program, task 1 and task 2 are established in ISIGHT. These two tasks are to calculate the total structural mass and intracavity sound pressure level. Finally, weighted computation of two computing results was carried out, getting the final optimization design results. Specific design program is shown in Fig.2. According to above program design flow, the optimization task design program is finished in iSIGHT.

\section{Numerical example}

The reinforced cylinder structure is shown in Fig.3. It imitates the aviation cockpit structure. This structure used one cylinder shell to simulate the fuselage cover, two end plates to simulate two end plates of cabin, internal plate structure to simulate floor, and three ring ribs and 12 longitudinal bars to simulate the fuselage. Two end plates were thin $(2 \mathrm{~mm})$ iron plate and other plates were thin $(1 \mathrm{~mm})$ iron plates. The rib structure was an L-shaped angle iron. The L-shaped side length of three ring angle iron was $3 \mathrm{~cm}$ and thickness was $3 \mathrm{~mm}$. The L-shaped side length of longitudinal angle iron was $2.5 \mathrm{~cm}$ and thickness was $3 \mathrm{~mm}$.

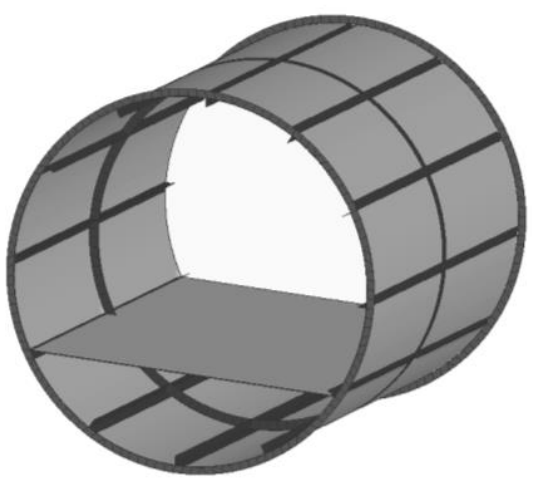

Fig. 3 Schematic of internal cylindrical structure

Based on above research program and software flow design, upper and lower limits of design variables were set in iSIGHT. The continuous secondary planning method +MOST combined algorithms were used as the optimization algorithm. Minimization of sound pressure in the field and total mass was taken as the objective function. According to analysis results of contributions of intracavity sound field, different substructures were used as the design variables. Sound and vibration analysis was implemented by VAOne. Then, the optimization calculation of sound field in the reinforced cylinder shell structural cavity under single point random force + external sound field mixed exciting conditions was accomplished. Final optimization results and analysis are introduced as follows:

According to analysis results of contribution of intracavity sound field under mixed exciting conditions, the front shroud, back shroud, 3\# plate, 17\# plate and 10\# plate which make the greatest 
contributions to intracavity noise were used as the design variables. Design range of the front and back shrouds was $0.001 \mathrm{~m} \sim 0.003 \mathrm{~m}$, and design range of rest plates was $0.0005 \mathrm{~m} \sim 0.002 \mathrm{~m}$. Final optimization results are shown in Table 1.

Table 1 Design variables before and after optimization

\begin{tabular}{|c|c|c|c|c|c|}
\hline & $\begin{array}{l}\text { front cover plate } \\
\qquad(\mathrm{m})\end{array}$ & $\begin{array}{c}\text { back cover plate } \\
(\mathrm{m})\end{array}$ & 17\#plate $(\mathrm{m})$ & 3\#plate $(\mathrm{m})$ & 10\#plate $(\mathrm{m})$ \\
\hline $\begin{array}{c}\text { Thickness before } \\
\text { optimization }\end{array}$ & 0.002 & 0.002 & 0.001 & 0.001 & 0.001 \\
\hline $\begin{array}{l}\text { Thickness after } \\
\text { optimization }\end{array}$ & 0.0012 & 0.0013 & 0.0007 & 0.00082 & 0.00093 \\
\hline
\end{tabular}

After the optimization, total mass of the reinforced cylinder shell structure decreased by $7.6 \%$ from the original $90.6 \mathrm{~kg}$ to $83.7 \mathrm{~kg}$. Comparison of sound pressure levels in the reinforced cylinder shell structural cavity before and after the optimization under mixed excitation conditions is shown in Fig.4. It can be seen that the average sound pressure level in the cavity reduced by $4.88 \mathrm{~dB}$ from the original $62.16 \mathrm{~dB}$ to $57.28 \mathrm{~dB}$. The structural size optimization in the middle and low frequency bands $(200 \mathrm{~Hz} 700 \mathrm{~Hz})$ influenced intracavity sound pressure level significantly. The maximum reduction of sound pressure level after optimization was about $7 \mathrm{~dB}$. Sound pressure level in high frequency band $(700 \mathrm{~Hz} 2000 \mathrm{~Hz})$ increased to some extent, but the amplitude was lower than $1 \mathrm{~dB}$, which influence the overall intracavity sound pressure level slightly. Fig.4 reveals that acoustic characteristics of reinforced cylinder shell structure after size optimization have been improved to some extent. The optimization is fruitful.

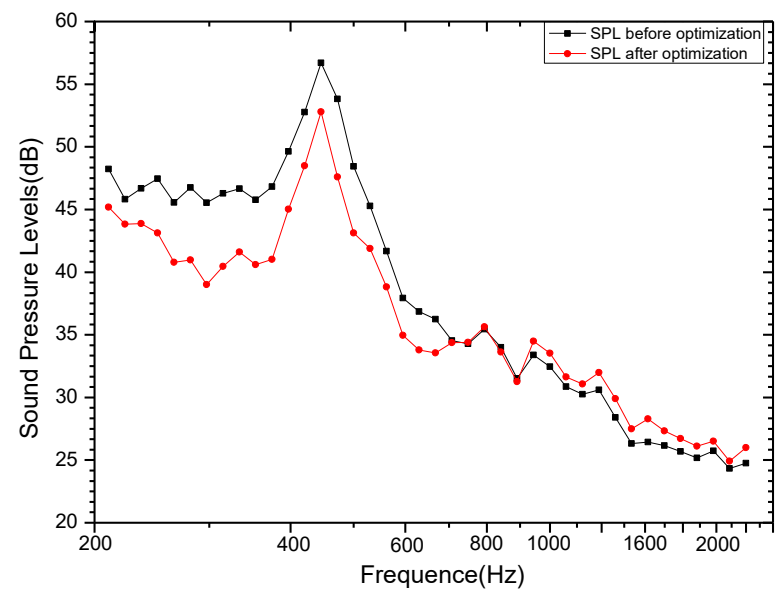

Fig.4 Comparison onSPL before and after the optimization under mixed excitation

\section{Conclusions}

This study discusses multi-objective optimization design of intracavity noise level in reinforced cylinder structure which is often used in various types of aircrafts. It establishes the optimization mathematical model, designs the total optimization flow and constructs the intracavity noise optimization design of cylinder structure. ISIGHT is integrated with VAOne for multi-objective optimization analysis. Design ranges of parameters are set, constraint conditions are determined, the optimization algorithm is chosen and the optimization operation is accomplished in the ISIGHT. Finally, the optimization outputs are analyzed and compared to those before optimization to verify effectiveness of the optimization algorithm. Computing results demonstrated that under different exciting conditions, intracavity sound pressure level is decreased to some extent after size 
optimization, especially in the low-frequency band. The reduction of maximum sound pressure level is $5 \sim 7 \mathrm{~dB}$. The acoustic characteristics of cavity structure are improved significantly. Therefore, the goal of noise reduction can be realized by reallocating thickness at different structural positions under relative fixed structural weight.

\section{Acknowledgements}

This work was financially supported by the National Natural Science Foundation of China (Grant .No. 11404205) and the Fundamental Research Funds for the Central Universities of Ministry of Education of China (Grant No. GK201703015).

\section{References}

[1] H. Denli, and J. Q. Sun: Structural-acoustic optimization of sandwich structures with cellular cores for minimum sound radiation. Journal of Sound and Vibration Vol. 301 (2007), p. 93

[2] S. Goo, S. Wang, and J. Kook: Topology optimization of bounded acoustic problems using the hybrid finite element-wave based method. Computer Methods in Applied Mechanics and Engineering Vol. 313 (2017), p. 834

[3] X. Wang, Y. Li, and Z. Ma: Robust optimization of structural-acoustic coupled system with random parameters. Aerospace Science and Technology Vol. 60 (2017), p. 48

[4] Y. Wang, X. Qin, and S. Huang: Structural-borne acoustics analysis and multi-objective optimization by using panel acoustic participation and response surface methodology. Applied Acoustics Vol. 116 (2017), p. 139

[5] S. Beigmoradi, H. Hajabdollahi, and Asghar Ramezani: Multi-objective aero acoustic optimization of rear end in a simplified car model by using hybrid Robust Parameter Design, Artificial Neural Networks and Genetic Algorithm methods. Computers \& Fluids Vol. 90 (2014), p. 123

[6] L. Chen, and Y. Zhang: A study on the application of material selection optimization approach for structural-acoustic optimization. Materials \& Design Vol. 52 (2013), p. 207

[7] Y. Zhang, H. Wu, and W. Jiang: Acoustic topology optimization of sound power using mapped acoustic radiation modes. Wave Motion Vol. 70 (2017), p. 90

[8] W. Li, Y.J. Jia, and Y. Wen: An improved collaborative optimization for multidisciplinary problems with coupled design variables. Advances in Engineering Software Vol. 102 (2016), p. 134

[9] B. Li, Y. Pang, and Y. Cheng: Collaborative optimization for ring-stiffened composite pressure hull of underwater vehicle based on lamination parameters. International Journal of Naval Architecture and Ocean Engineering Vol. 9 (2016), p. 1

[10] L. Yang, J. Qi, and S. Li: Collaborative optimization for train scheduling and train stop planning on high-speed railways. Omega vol. 64 (2016), p. 57 